\title{
DUCTO VENOSO: DA ANATOMIA À AVALIAÇÃO DO BEM-ESTAR FETAL
}

\author{
DUCTUS VENOSUS: FROM THE ANATOMY TO FETAL WELL BEING EVALUATION
}

\author{
Geraldo Duarte ${ }^{1}$, Alessandra Cristina Marcolin², Gerson Cláudio Crott ${ }^{3}$, \\ Carla Vitola Gonçalves ${ }^{2}$ \& Aderson Tadeu Berezowski ${ }^{1}$
}

\begin{abstract}
'Docentes; ${ }^{2}$ Alunas de Pós-graduação do Setor de Patologia Obstétrica. Departamento de Ginecologia e Obstetrícia (RGO) da Faculdade de Medicina de Ribeirão Preto-USP. (FMRP-USP). ${ }^{3}$ Médico do Departamento de Puericultura e Pediatria da FMRP-USP. CorRespondênCIA: Geraldo Duarte. Hospital das Clínicas da Faculdade de Medicina de Ribeirão Preto - $8^{\circ}$ andar. Avenida Bandeirantes, 3900. Ribeirão Preto - São Paulo. CEP-14048-900. Fone: (16) 602-2588. e-mail: gduarte@fmrp.usp.br
\end{abstract}

DUARTE G; MARCOLIN AC; CROTT GC; GONÇALVES CV \& BEREZOWSKIAT. Ducto venoso: da anatomia à avaliação do bem-estar fetal. Medicina, Ribeirão Preto, 34: 301-307, jul./dez. 2001.

RESUMO: O ducto venoso é a continuação da veia umbilical com a veia cava inferior, e ele, posteriormente ao nascimento, oblitera-se, formando o ligamento venoso. Por meio dele, o sangue rico em oxigênio, proveniente da veia umbilical, chega ao átrio direito e a partir daí, pelo forame oval, ganha o átrio esquerdo e a circulação sistêmica, favorecendo o fluxo para órgãos vitais como o cérebro fetal.

Em obstetrícia, a ultra-sonografia Doppler tem sido amplamente utilizada para se examinar o sistema arterial fetal. Recentemente, mais atenção tem sido dispensada ao sistema venoso. $O$ estudo das ondas de velocidade de fluxo venoso pode desempenhar um papel importante na avaliação do bem-estar fetal, uma vez que a velocimetria do ducto venoso pode estar alterada na vigência de patologias fetais. A característica mais interessante é a redução ou fluxo reverso durante a contração atrial, comumente encontrada em fetos com defeitos cardíacos congênitos, arritmias, transfusão fetofetal grave e restrição de crescimento intra-útero.

Este texto tem a finalidade de revisar as características anatômicas e funcionais do ducto venoso fetal, bem como a importância de sua avaliação ultra-sonográfica pré-natal.

UNITERMOS: Ducto Venoso. Gravidez. Avaliação. Feto.

\section{INTRODUÇÃO}

No passado, acreditou-se que, com a introdução da cardiotocografia basal e do teste de ocitocina na avaliação pré-natal do bem-estar fetal, seria possível detectar todas as situações de hipoxia ou asfixia às quais o feto estivesse submetido. Porém, apesar de ambos estarem associados a baixas taxas de resultados falso-negativos, apresentam elevadas taxas de resultados falso-positivos, tornando-os instrumentos limitados para detectar o feto comprometido, no momento do exame ${ }^{(1)}$.

Com os avanços tecnológicos da ultra-sonografia, como o exame em tempo real, a escala de tons de cinza e a alta definição, verificou-se notável evolução no estudo da anatomia fetal, na análise da idade gestacional e na avaliação da vitalidade fetal. Em 1980, Manning et al. propuseram a utilização de cinco variáveis biofísicas do feto como forma de prever o resultado perinatal, sendo atribuída pontuação específi- 
ca a cada uma delas. Foram considerados os movimentos somáticos, os movimentos respiratórios, o tônus fetal e o volume de líquido amniótico ${ }^{(2)}$. Outro sistema de avaliação, proposto por Vintzileos et al. (1983), incluía o grau de maturidade, placentária como variável biofísica ${ }^{(3)}$. Em ambos os protocolos, inicia-se a avaliação fetal com a cardiotocografia. Portanto, considera-se que o perfil biofísico do feto seja uma combinação de marcadores agudos e crônicos, de comprometimento do bem-estar fetal,sujeita a situações que dificultam sua avaliação plena, como administração de drogas à mãe, infecção maternofetal, rotura prematura de membranas amnióticas, influência da variação diurna na periodicidade das variáveis analisadas e a idade gestacional ${ }^{(4)}$.

Essas limitações impuseram a busca e a adaptação de outros métodos para a avaliação do bemestar fetal e que preenchessem alguns requisitos, tais como: a elevada taxa de reprodutibilidade, baixo custo, fácil realização, não ser invasivo e apresentar elevadas taxas de sensibilidade e especificidade ${ }^{(5)}$. A utilização do método Doppler, na avaliação do bem-estar fetal, aproximou-se bastante dos pré-requisitos acima propostos $^{(6)}$. Além de complementar a avaliação de vitalidade fetal, proporciona melhor avaliação de complicações da gestação, como retardo de crescimento intra-uterino, outras formas de sofrimento fetal, que resultam de hipoxemia ou asfixia fetal, e anomalias estruturais do feto e de seus anexos ${ }^{(4)}$.

Com o método Doppler, surgiu o conceito de Centralização Fetal, que passou a ser considerado como o mais precoce e fidedigno sinal de comprometimento hipóxico fetal ${ }^{(7,8)}$. A hipoxemia e a acidose fetais produzem uma redistribuição protetora do débito cardíaco, diminuindo o fluxo sangüíneo a órgãos - como pulmões, intestinos - musculatura e ossos, permitindo maior fluxo para o cérebro, coração e suprarenais. As variáveis biofísicas permanecem normais enquanto o mecanismo da centralização fetal for suficiente para manter o bom funcionamento dos órgãos vitais. Quando ele for insuficiente na manutenção da função dos órgãos nobres, freqüentemente observam-se alterações de perfil biofísico fetal ${ }^{(5)}$.

A análise dos vasos venosos fetais, utilizando o método Doppler, pode demonstrar modificações adaptativas precoces, que, talvez, antecedam as alterações vasculares arteriais, identificando precocemente os primeiros sinais de insuficiência cardíaca fetal, relativos a hipoxia ${ }^{(9)}$. Com esses achados, mais atenção tem sido dispensada ao estudo do sistema venoso fetal, em especial ao ducto venoso $(\mathrm{dv})^{(10)}$.

\section{ANATOMIA}

$\mathrm{O} d v$ é um pequeno vaso que aparece no embrião humano em torno do $30^{\circ}$ dia de vida, ou com $6,5 \mathrm{~mm}$ de comprimento crânio-nádega ${ }^{(11)}$, a partir do momento em que a drenagem sangüínea da placenta, que até então era feita através de duas veias umbilicais, passa a ser realizada apenas pela veia umbilical esquerda, com regressão da direita ${ }^{(12)}$. Essa mudança impõe a necessidade de um controle melhor do fluxo sangüíneo, na tentativa de manter um balanço pressórico e adequado, entre a veia umbilical e o retorno venoso para o coração, surgindo alterações da pressão e da morfologia vascular. O dv surge de um sinusóide hepático mais proeminente e que une diretamente a veia umbilical à veia cava inferior. Precocemente, já pode ser notada pequena projeção na parede do $d v$, projeção que se desenvolve gradualmente, originando estrutura músculoelástica especializada, de forma triangular, com a base disposta ao longo da parede e com o ápice voltado para o lúmen, sugerindo a existência de um esfíncter e de sua possível capacidade funcional ${ }^{(11)}$.

$\mathrm{O} d v$ continua seu desenvolvimento a partir do final do período embrionário até o termo, crescendo linearmente cerca de 10 vezes, tanto em comprimento quanto em diâmetro. No termo, costuma ter aproximadamente $2 \mathrm{~cm}$ de comprimento por $2 \mathrm{~mm}$ de diâmetro, possuindo rica rede de fibras elásticas e musculares, dispostas em camadas ao longo das paredes ${ }^{(13)}$, menos na área do esfíncter, e, entre elas e o endotélio, há uma camada especial de fibras musculares longitudinais ${ }^{(11)}$. Com relação à inervação, diversas são as fontes. Fibras de ambos os nervos frênicos atingem a parede superior do vaso e se distribuem para a região do esfíncter, podendo ser vistas em embriões de 19 $\mathrm{mm}$. Fibras vagais descem pelo ligamento hepatogástrico e se distribuem ao longo das paredes do dv, atingindo a junção deste com a veia umbilical na região do esfíncter, assim como fibras ascendentes do plexo celíaco, já vistas em embriões de $16 \mathrm{~mm}^{(14)}$.

$\mathrm{O} d v$ origina-se a partir da porção umbilical da veia porta esquerda, continuando, posterior e cranialmente, sobre a porção superior do ligamento hepatogástrico. No período pós-natal, torna-se o ligamento venoso $^{(12)}$.

\section{FISIOLOGIA}

Funcionando exclusivamente na circulação fetal, o dv torna-se funcionalmente impérvio após o nasci- 
mento, na maioria dos mamíferos. No intuito de se estudar o fechamento pós-natal do dv, foram realizados vários estudos experimentais, utilizando-se exames contrastados em veia umbilical de fetos vivos de ovelhas, retirados do útero e mantidos ligados à placenta pelo cordão umbilical. Desse modo, Barclay et al.(1942) observaram a existência de padrão oscilatório do contraste no dv, com aparecimento e desaparecimento alternados do mesmo, sugerindo uma tendência de relaxamento e constricção sucessivos, até a oclusão final. Esses pesquisadores acreditam que o $\mathrm{dv}$ se fecha completamente, a partir da região esfincteriana, em associação à diminuição do fluxo sangüíneo umbilical e com a contribuição de espasmo muscular ${ }^{(15)}$. Resultados semelhantes também são citados por Meyer et al.(1966), referindo que o fechamento do dv inicia-se com a queda pós-natal da pressão, na veia umbilical (de 20 - $30 \mathrm{mmHg}$ para $7-8 \mathrm{mmHg}$ ), e conseqüente redução do calibre da mesma, retraindo as bordas do orifício de entrada do ducto. Para esses autores, o processo contaria, também, com o auxílio das fibras musculares de sua parede, completando o fechamento funcional. Em seguida, ocorreria a obliteração orgânica, a partir do tecido de revestimento de sua entrada, estreitada através da proliferação de tecido conjuntivo, frouxo, rico em células e pobre em fibras. Na evolução, o tecido proliferado oclui totalmente a abertura residual do vaso entre o $15^{\circ}$ e o $20^{\circ}$, dias de vida extra-uterina ${ }^{(13)}$.

$\mathrm{O} d v$ atua, transmitindo sangue bem oxigenado, proveniente da veia umbilical $(\mathrm{vu})$, para o coração, desviando-o da circulação hepática ${ }^{(16)}$. Experimentos, em animais, mostram que cerca de 50 - 60\% do sangue umbilical contorna o fígado através do $\mathrm{dv}$, drenando diretamente na veia cava inferior. A partir daí, o sangue ganha o átrio direito e, através do forâmen oval, atinge o lado esquerdo do coração e a circulação sistêmica. Logo, sangue rico em oxigênio supre órgãos vitais como o cérebro, as supra-renais e o coração. $\mathrm{O}$ restante do sangue venoso umbilical passa, através de sinusóides hepáticos, sendo drenado para a veia cava inferior através das veias hepáticas ${ }^{(17)}$. A pressão, na vu fetal, é de 4 - $5 \mathrm{mmHg}$ em torno da $26^{\mathrm{a}}$ semana de gestação, pressão essa que age como força motriz capaz de impulsionar o sangue para o $\mathrm{dv}^{(16)}$.

\section{AVALIAÇÃO ULTRA-SONOGRÁFICA}

O dv é um vaso cuja visualização ultra-sonográfica pode ser difícil em gestações de primeiro trimestre. Pode ser visualizado tanto no plano longitudinal, em corte sagital do tronco fetal, como em corte transverso do abdome fetal ${ }^{(18)}$. Tomando-se o volume de amostra sobre sua origem, a partir do "sinus" portal, ondas com padrões característicos podem ser visualizadas. A melhora dos equipamentos ultrasonográficos, providos de Doppler colorido e pulsátil, tornou mais fácil a identificação de tal estrutura ${ }^{(19)}$.

Ondas de velocidade de fluxo do dv são caracterizadas por um pico sistólico, um diastólico e um "nadir" durante a contração atrial, sem componente de fluxo reverso após (Figura 1). A ausência de fluxo

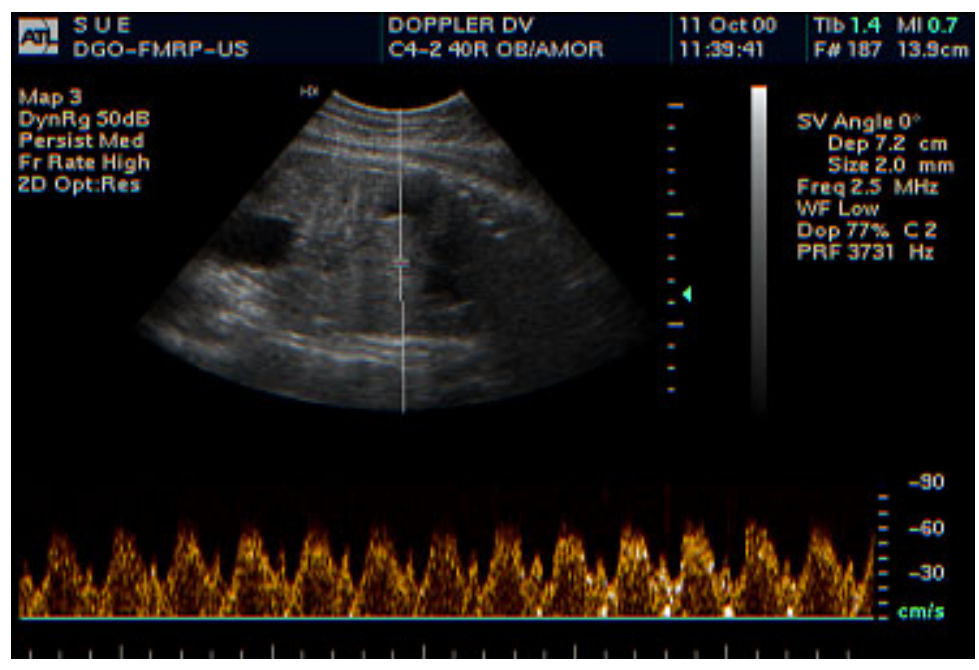

Figura 1: Ondas normais de velocidade de fluxo do ducto venoso fetal. Podemos observar seu padrão bifásico, com um pico sistólico, um pico diastólico e um nadir durante a contração atrial. 
reverso no $\mathrm{dv}$ pode ser o resultado indireto do gradiente de pressão entre a veia umbilical e o átrio direito ${ }^{(10)}$. Essas características denotam uma onda de padrão bifásico, podendo ser registradas em torno de 8 - 9 semanas de gestação. Com 11-12 semanas, os componentes sistólico e diastólico tornam-se claramente visíveis, atingindo picos de velocidade de até $30 \mathrm{~cm} / \mathrm{s}$. A velocidade média de fluxo desse vaso é três vezes maior que aquela observada na veia cava inferior e umbilical ${ }^{(10,19)}$.

A avaliação do fluxo do dv, realizado por Montenegro et al.(1997), utilizando o método Doppler, em gestantes de primeiro trimestre, não mostrou variações nos valores de velocidade do pico sistólico, do pico diastólico e nem da velocidade média de fluxo. Do mesmo modo, os valores obtidos durante a contração atrial e a relação S/D mantiveram-se constantes. Por outro lado, houve redução na frequiência dos batimentos cardíacos da $10^{\text {a }}$ a $13^{\text {a }}$ semanas de gestação, fato este atribuído à maturação do sistema nervoso parassimpático ${ }^{(20)}$. Huisman et al.(1992) mostraram correlação positiva entre a idade gestacional e a velocidade média, o pico sistólico e o diastólico do dv, com aumento de todos os itens citados, embora, mantendo a relação S/D constante. Esses autores postularam que muitos fatores podem estar envolvidos nessas alterações, tais como o aumento do volume de fluxo através do ducto, aumento da complacência cardíaca e a redução da pós-carga, sendo esta última resultado da diminuição da resistência vascular placentária, com o evoluir da gestação ${ }^{(20,21)}$.

Diversas anomalias de retorno venoso devido à agenesia do dv já foram descritas na literatura( ${ }^{(22,23)}$, ao lado de casos em que a agenesia do mesmo não comprometeu a hemodinâmica fetal. Em tais casos, as veias hepáticas assumiram a função do vaso ausente $^{(17)}$.

Vários fatores têm sido envolvidos nas mudanças que ocorrem no fluxo do dv, como, por exemplo, o comportamento fetal. Em estudo realizado por Huisman et al.(1994), foi demonstrado que, durante o período de repouso fetal, ocorre redução de aproximadamente $30 \%$ na velocidade de fluxo no pico sistólico, no pico diastólico e na velocidade média, sugerindo a redução do volume de fluxo durante aquele período. Porém, comparando-o com o período de sono REM (Rapid Eye Movement), foi visto, neste último, um aumento no fluxo através do dv, sendo esse achado consistente com relatos que mostram fluxo aumenta- do no nível do forame oval e da valva mitral durante o período de sono. Isso é extremamente benéfico, visto ser necessário fluxo cerebral maior durante a fase mais ativa do feto. Talvez essas alterações ocorrem devido a uma redistribuição do sangue venoso umbilical durante os diferentes períodos de atividade fetal ${ }^{(24)}$.

Em 1998, Matias et al., correlacionaram alterações nas ondas de velocidade de fluxo no dv, em fetos, entre 10-14 semanas, com alterações cromossômicas e translucência nucal aumentada. Foi observada ausência ou fluxo reverso durante a contração atrial. Em uma pequena porcentagem de fetos geneticamente normais $(3,1 \%)$ e com translucência nucal aumentada, esse detalhe também foi visto, porém os mesmos apresentavam algum defeito cardíaco. Esse achado é concorde com dados de outros autores, demonstrando que uma grande proporção de fetos com ou sem anomalias cromossômicas, mas com translucência nucal aumentada, apresentam anomalias cardíacas ou de grandes vasos. In vivo, evidências de falência cardíaca estão relacionadas a alterações na onda de velocidade de fluxo do dv. Assim, sua avaliação pode ser útil no screening de anormalidades cardíacas, combinando idade materna e translucência nucal aumentada em gestações de 10-14 semanas $^{(25)}$. Em 1999, Murta et al. avaliaram fetos com translucência nucal aumentada, submetidos à cariótipo entre 10-14 semanas de idade gestacional, demonstrando novamente os achados acima citados. As alterações da onda do $\mathrm{dv}$ estariam relacionadas, no primeiro trimestre, à imaturidade ventricular, com prejuízo da função diastólica, ao lado de uma pós-carga cardíaca aumentada pela maior resistência placentária ${ }^{(26)}$.

Medidas de velocidade de fluxo com ultra-sonografia Doppler, aferidas em fetos com grave retardo de crescimento intra-útero e em gestações gemelares, também têm mostrado alterações. Em 1998, Tchirikov et al. sugeriram que esse evento possa decorrer de um aumento na resistência vascular hepática e conseqüente aumento de fluxo no dv, fluxo que, geralmente, acompanha essas condições, como mecanismo compensatório para manter o suprimento de oxigênio adequado para os órgãos vitais ${ }^{(27)}$. A ausência ou fluxo reverso, na contração atrial, pode representar um fenômeno tardio no feto, com restrição de crescimento e sofrimento fetal crônico ${ }^{(28)}$. Ao lado desses achados, foi observado que a hipoxemia e a infusão de catecolaminas causam aumento da relação fluxo dv/ fluxo vu(29). 
Em 1995, Hecher et al. estudaram fetos não comprometidos e fetos comprometidos, sendo estes últimos aqueles nascidos por cesárea, dentro de dez dias do último exame Doppler, devido a cardiotocografia alterada, piora do quadro de doença hipertensiva da gestação ou óbito fetal. Os autores avaliaram ondas de velocidade de fluxo das artérias umbilicais, aorta torácica descendente, artéria cerebral média, valvas tricúspide e mitral, ducto venoso, veia cava inferior e veia hepática direita, procurando estabelecer a cronologia de aparecimento das alterações arteriais e venosas em fetos comprometidos, grupo que mostrou alterações significativas tanto nas ondas arteriais como nas venosas. Dos fetos comprometidos, aqueles com alteração do perfil biofísico mostraram alterações significativas em todos os vasos venosos, enquanto as diferenças no lado arterial foram menos pronunciadas. No ducto venoso, foram encontrados índices de velocidade de fluxo elevados, além de ausência ou fluxo reverso durante a contração atrial, relacionados ao aumento da pós-carga ou à falência miocárdica secundária à deterioração pela redistribuição arterial fetal $^{(18)}$ (Figura 2).

A análise do dv pode ser utilizada como um fator preditor de hipoxemia fetal, como demonstrado em estudo realizado por Rizzo et al.(1996), no qual, a redução na velocidade de fluxo, encontrada durante contração atrial, parece refletir aumento das resistências periférica e placentária e redução da contratilidade cardíaca, aumentando a pressão diastólica final ${ }^{(30)}$. Achados semelhantes foram encontrados por Hecher et al.(1995), que demonstraram a associação dos pa- râmetros alterados com acidose fetal, confirmada por cordocentese $^{(31)}$.

A investigação do fluxo sangüíneo venoso também pode ajudar na identificação do comprometimento fetal por complicações com o cordão umbilical. Em relato de caso citado por Bäz et al.(1999), a avaliação Doppler do dv de feto com circular de cordão, mostrou ondas com fluxo reverso durante a contração atrial e índice de pulsatilidade marcadamente aumentado. A isquemia relativa, provocada pela tração do cordão, em longo prazo, reduz o suprimento sangüíneo fetoplacentário, levando à redistribuição do fluxo sangüíneo fetal antes que o fluxo venoso seja afetado. Logo, os achados no ducto venoso indicariam pressão ventricular diastólica final, aumentada devido à falência cardíaca relacionada a hipoxemia e acidose fetais, comprovadas pela gasometria do cordão ${ }^{(32)}$.

Outra contribuição do método Doppler do dv seria na isoimunização, em que um estado hiperdinâmico, conseqüente à anemia, leva a aumento na velocidade de fluxo, embora, não apresente poder discriminatório entre os graus de severidade do processo anêmico ${ }^{(33)}$.

Por fim, nova tendência, na literatura, vem mostrando que o fluxo sangüíneo que passa através do dv, em gestações normais, talvez, não seja tão intenso, quando comparado ao fluxo hepático como citado até hoje. Em trabalho realizado por Kiserud et al.(2000), mostrou-se que apenas 20-30\% do fluxo umbilical atinge o dv durante a segunda metade da gestação, ao lado dos 70-80\% que acabam perfundindo o fígado. Esses achados podem indicar que a demanda de fluxo através do dv é modesta durante a gravidez normal,

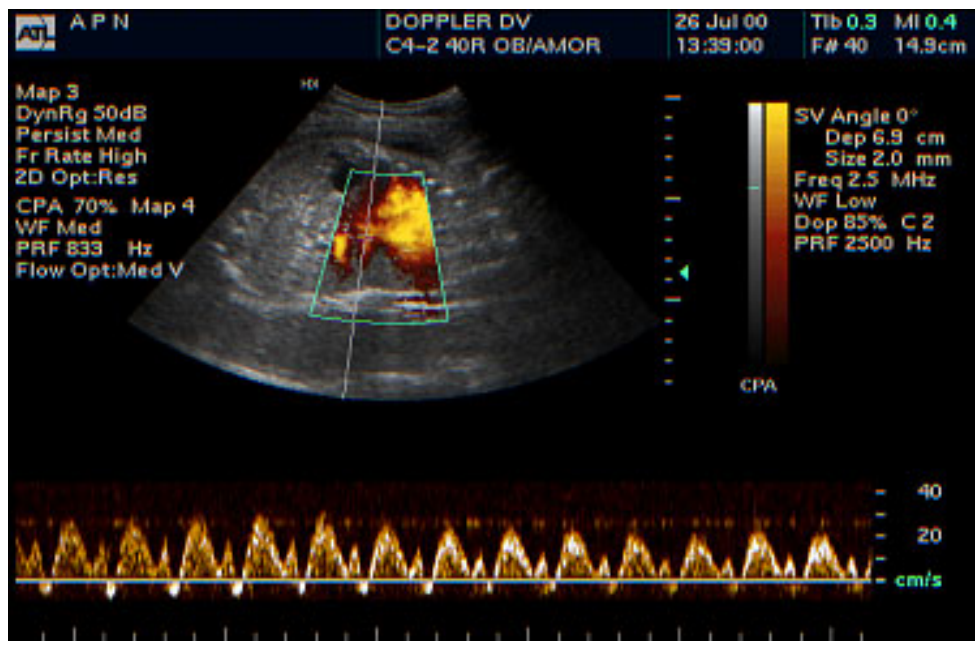

Figura 2: Padrão anormal de ondas de velocidade de fluxo do ducto venoso fetal. Podemos observar o fluxo reverso durante a contração atrial. 
sem comprometer o desenvolvimento fetal, porém, isso não significa que não haja um fluxo aumentado em circunstâncias especiais, como alterações hemodinâmicas agudas e hipoxemia, como já citado em diversos trabalhos, na literatura. Também, não se pode es- quecer que diferenças de resultados podem ser decorrentes do uso de diferentes métodos, erros nas medidas dos diâmetros de pequenos vasos (como é o caso do $d v)$ e diferenças entre as espécies utilizadas em avaliações realizadas previamente ${ }^{(34)}$.

DUARTE G; MARCOLIN AC; CROTT GC; GONÇALVES CV \& BEREZOWSKI AT. Ductus venosus: From the anatomy to fetal well being evaluation. Medicina, Ribeirão Preto, 34: 301-307, july/dec. 2001.

ABSTRACT: The ductus venosus is a blood vessel unique to the fetal circulation which functions as a shunt between the umbilical vein and inferior vena cava. Well-oxygenated blood from the umbilical vein will course almost directly through the ductus venosus towards the foramen ovale and left heart favoring flow to vital organs as the fetal brain. It becomes functionally impervious to blood just after birth, in the majority of mammals.

In obstetrics, Doppler ultrasound scan has largely been used to examine the fetal arterial system. Recently, more attention has been paid to the venous system. Assessment of venous flow velocity waveforms can play an important role in the surveillance of fetal well-being. The ductus venosus velocimetry can be altered in fetal diseases. The most striking feature is the reduced or reversed flow during atrial contraction commonly found in fetuses with congenital heart defects, arrhythmias, twin-twin transfusion syndrome and serious intrauterine growth restriction.

This text includes a review of the anatomic and physiologic characteristics of fetal ductus venosus and the importance of its evaluation by prenatal ultrasound.

UNITERMS: Ductus Venosus. Pregnancy. Fetus. Evaluation.

\section{REFERÊNCIAS BIBLIOGRÁFICAS}

1 - EVERTSON LR; GAUTHIER RJ \& SCHIFRIN BS. Antepartum fetal heart rate testing: evolution of the nonstress test. Am J Obstet Gynecol 133: 29-33, 1979.

2 - MANNING FA; PLATT LD \& SIPOS L. Antepartum fetal evaluation: development of a fetal biophysical profile score. Am $\mathbf{J}$ Obstet Gynecol 136: 787-795, 1980.

3 - VINTZILEOS AM; CAMPBELL WA \& INGARDIA CJ. The fetal biophysical profile and its predictive value. Obstet Gynecol 62: 271-278, 1983.

4 - VINTZILEOS AM; CAMPBELL WA \& RODIS JF. Avaliação prénatal por ultra-sonografia: o perfil biofísico fetal. In: CALLEN $\mathrm{PW}$, ed. Ultra-sonografia em obstetrícia e ginecologia, $3^{\mathrm{a}}$ ed, Editora Guanabara Koogan, Rio de Janeiro, p.473-487, 1996.

5 - BEREZOWSKI AT \& CUNHASP. Perfil biofísico fetal. In: CUNHA SP \& DUARTE G, eds. Gestação de alto risco, MEDSI Editora Médica e Científica, Rio de Janeiro, p.447-458, 1998.

6 - SOOTHILL PW; AJAYI RA; CAMPBELL S \& NICOLAIDES KH. Prediction of morbidity in small and normaly grown fetuses by fetal heart rate variability, biophysical score and umbilical artery doppler studies. Br J Obstet Gynecol 100: 742-745, 1993.

7 - CAMPBELL S; GRIFFIN DR \& PEARCE JM. New Doppler technique for assessing utero placental blood flow. Lancet 1: 675-677, 1983.
8 - CAMPBELL S; PEARCE MF \& HACKETT G. Qualitative assessment of uteroplacental blood flow: early screening test for high-risk pregnancies. Obstet Gynecol 68: 649-653, 1986.

9 - GUDMUNSSON G; TULZER G; HUHTA JC \& MARSAL K. Venous doppler in the fetus with absent end-diastolic in the umbilical artery. Ultrasound Obstet Gynecol 7: 262-267, 1996.

10 - MARI G; UERPAIROJKIT B \& COPEL JA. Abdominal venous system in the normal fetus. Obstet Gynecol 86: 729-733, 1995.

11 - CHACKO AW \& REYNOLDS SRM. Embryonic development in the human of the sphincter of ductus venosus. Anat Rec 115: 151-173, 1953.

12 - CHINN DH; FILLY RA \& CALLEN PW. Ultrasonic evaluation of fetal umbilical and hepatic vascular anatomy. Radiology 144: 153-157, 1982

13 - MEYER WW \& LIND J. The ductus venosus and the mechanism of its closure. Arch Dis Child 41: 597-605, 1966.

14 - PEARSON AA \& SAUTER RW. Observations on the phrenic nerves and the ductus venosus in human embryos and fetuses. Am J Obstet Gynecol 15: 560-565, 1971.

15 - BARCLAY AE; FRANKLIN KJ \& PRICHARD MM. The mechanism of closure of the ductus venosus. Br J Radiol 15: 6671, 1942. 
16 - KISERUD T; EIK-NES SH; BLAAS HGK \& HELLEVIK LR. Ultrasonografic velocimetry of the fetal ductus venosus. Lancet 338: 1412-1414, 1991.

17 - GEMBRUH U; BASCHAT AA; CALIEBE A \& GORTNER L. Prenatal diagnosis of ductus venosus agenesis: a report of two cases and review of the literature. Ultrasound Obstet Gynecol 11: 185-189, 1998.

18 - HECHER K; CAMPBELL S; DOYLE P; HARRINGTON K \& NICOLAIDES K. Assessment of compromise by doppler ultrasound investigation of the fetal circulation. Circulation 91: 129-138, 1995.

19 - HUISMAN TWA; WLADIMIROFF JW \& STEWART PA. The normal early circulation: a doppler ultrasound study. In: KURJAC A, ed. An atlas of transvaginal color doppler: the current state of the art, The Parthenon Publishing Group, New York, p.43-49, 1994.

20 - MONTENEGRO N; MATIAS A; AREIAS JC \& BARROS H. Ductus venosus revisited: a doppler blood flow evaluation in the first trimester of pregnancy. Ultrasound Med Biol 23: 171-176, 1997.

21 - HUISMAN TWA; STEWART PA \& WLADIMIROFF JW. Ductus venosus blood flow velocity waveforms in the human fetus - a doppler study. Ultrasound Med Biol 18: 33-37, 1992.

22 - MOORE L; TOI A \& CHITAYAT D. Anormalities of the intraabdominal fetal umbilical vein: reports of four cases and a review of the literature. Ultrasound Obstet Gynecol 7: 21-25, 1996

23 - JÖRGENSEN C \& ANDOLF E. Four cases of absent ductus venosus: three in combination with severe hydrops fetalis. Fetal Diagn Ther 9: 395-399, 1994.

24 - HUISMAN TWA; BREZINKA C; STEWART PA; STIJNEN T \& WLADIMIROFF JW. Ductus venosus flow velocity waveforms in relation to fetal behavioural states. $\mathbf{B r} \mathbf{J}$ Obstet Gynaecol 101: 220-224, 1994.

25 - MATIAS A; GOMES C; FLACK N; MONTENEGRO N \& NICOLAIDES KH. Screening for chromosomal abnormalities at $10-14$ weeks: the role of ductus venosus blood flow. Ultrasound Obstet Gynecol 12: 380-384, 1998.
26 - MURTA CG; MORON AF \& de AVILA MA. Detection of functional changes of the fetal heart in the first trimester of gestation. Arq Bras Cardiol 72: 739-750, 1999.

27 - TCHIRIKOV M; RYBAKOWSKI C; HÜNEKE B \& SCHRÖDER HJ. Blood flow through the ductus venosus in singleton and multifetal pregnancies and in fetuses with intrauterine growth retardation. Am J Obstet Gynecol 178: 943-949, 1998.

28 - OZCAN T; SBRACIA M; D'ANCONA RL; COPEL JA \& MARI G. Arterial and venous Doppler velocimetry in the severely growth-restricted fetus and associations with adverse perinatal outcome. Ultrasound Obstet Gynecol 12: 39-44, 1998.

29 - TCHIRIKOV M; EISERMANN K; RYBAKOWSKI C \& SCHRÖDER HJ. Doppler ultrasound evaluation of ductus venous blood flow during acute hipoxemia in fetal lambs. Ultrasound Obstet Gynecol 11: 426-231, 1998.

30 - RIZZO G; CAPPONI A; TALONE PE; ARDUINI D \& ROMANINI C. Doppler indices from inferior vena cava and ductus venosus in predicting $\mathrm{pH}$ and oxygen tension in umbilical blood at cordocentesis in growth-retarded fetuses. Ultrasound Obstet Gynecol 7:401-410, 1996.

31 - HECHER K; SNIJDERS R; CAMPBELL S \& NICOLAIDES K. Fetal venous, intracardiac, and arterial blood flow measurements in growth retardation: Relationship with fetal blood gases. Am J Obstet Gynecol 173: 10-15, 1995.

32 - BÄZ E; ZIKULNIG B; HACKELÖER J \& HECHER K. Abnormal ductus venosus blood flow: a clue to umbilical cord complication. Ultrasound Obstet Gynecol 13: 204-206, 1999.

33 - HECHER K; SNIJDERS R; CAMPBELL S \& NICOLAIDES K. fetal venous, arterial, and intracardiac blood flows in red blood cell isoimmunization. Obstet Gynecol 85: 122-127, 1995.

34 - KISERUD T; RASMUSSEN S \& SKULSTAD S. Blood flow and degree of shunting through the ductus venosus in the human fetus. Am J Obstet Gynecol 182: 147-153, 2000.

Recebido para publicação em 25/02/2001

Aprovado para publicação em 22/07/2001 